\title{
GSH Attenuates Organ Injury and Improves Function after Transplantation of Fatty Livers
}

\author{
S. Pratschke M.K. Angele U. Grützner A.Tufman M. Bilzer F. Loehe \\ K.-W. Jauch R.J. Schauer \\ Department of Surgery, Klinikum Grosshadern, Ludwig-Maximilians-University, Munich, Germany
}

\section{Key Words}

Fatty liver $\cdot$ Glutathione $\cdot$ Organ injury $\cdot$ Transplantation

\begin{abstract}
Ischemia-reperfusion injury (IRI) is increased after transplantation of steatotic livers. Since those livers are increasingly used for transplantation, protective strategies must be developed. Reactive oxygen species (ROS) play a key role in hepatic IRI. In lean organs, glutathione (GSH) is an efficient scavenger of ROS, diminishing IRI. The aim of this study was to evaluate whether GSH also protects steatotic allografts from IRI following transplantation. Fatty or lean livers were explanted from 10-week-old obese or lean Zucker rats and preserved (obese $4 \mathrm{~h}$, lean $24 \mathrm{~h}$ ) in hypothermic University of Wisconsin solution. Arterialized liver transplantation was then performed in lean syngeneic Zucker rats. Recipients of fatty livers were treated with GSH $(200 \mu \mathrm{mol} / \mathrm{h} / \mathrm{kg})$ or saline during reperfusion $(2 h, n=5)$. Parameters of hepatocellular damage and bile flow were measured. Transplantation of steatotic livers enhanced early reperfusion injury compared to lean organs as measured by increased aspartate aminotransferase, alanine aminotransferase, and lactate dehydrogenase plasma levels. Bile flow was also reduced in steatotic grafts. Intravenous administration of GSH effectively decreased liver damage in fatty allografts and resulted in im-
\end{abstract}

\section{KARGER}

Fax +41613061234 E-Mail karger@karger.ch www.karger.com
(C) 2010 S. Karger AG, Base

$0014-312 \mathrm{X} / 10 / 0451-0013 \$ 26.00 / 0$

Accessible online at:

www.karger.com/es proved bile flow. Intravenous application of GSH effectively reduces early IRI in steatotic allografts and improves recovery of these marginal donor organs following transplantation.

Copyright $\odot 2010$ S. Karger AG, Basel

\section{Introduction}

In an attempt to overcome the discrepancy between liver organ availability and demand, livers with poor organ quality are increasingly accepted for transplantation. In deceased organ donors, the prevalence of steatosis ranges from 13 to $28 \%$, approaching $50 \%$ when sensitive histological techniques are used $[1,2]$. Demographic data suggest a strong increase of obesity in the population, which may have a negative impact on the quality of harvested allografts used for liver transplantation [3].

Several human studies have demonstrated an increased ischemia-reperfusion injury (IRI) following transplantation of steatotic liver grafts as evidenced by

S.P. and M.K.A. contributed equally to the manuscript.
Martin Angele, MD, Department of Surgery

Klinikum Grosshadern, Ludwig-Maximilians-University Munich

Marchioninistrasse 15, DE-81377 Munich (Germany)

Tel. +49897095 0, Fax +49897095 5674

E-Mail Martin.Angele@med.uni-muenchen.de 
elevated plasma liver enzyme levels and delayed organ function $[3,4]$. Zucker rats homozygotic for a leptine-receptor gene defect (fa/fa) develop macrovesicular hepatic steatosis. Studies have described increased reperfusion injury in fa/fa Zucker rats compared to animals with healthy livers following warm and cold ischemia $[5,6]$. Therefore, fa/fa Zucker rats may represent a suitable animal model of IRI following transplantation of non-alcoholic fatty livers. Therefore, those rats may represent a suitable animal model of reperfusion injury following transplantation of non-alcoholic fatty livers.

Although the precise sequence of events leading to IRI in liver transplants has not been completely described, the generation of reactive oxygen species (ROS) and disturbances of the hepatic microcirculation may play key roles in the mechanisms following reperfusion injury [7, 8]. Administration of the antioxidant glutathione (GSH) has been shown to attenuate ischemia-reperfusion injury following warm ischemia or liver transplantation in healthy organs $[9,10]$. However, the effect of GSH on hepatocellular damage caused by reperfusion of transplanted fatty livers remains largely unknown. Recent work has demonstrated differences in the cellular and humoral response in healthy and diseased livers following exposure to ischemia and reperfusion [11].

The purpose of the present study is to test the efficacy of post-ischemic intravenous GSH administration in preventing early reperfusion injury after transplantation of fatty livers. Despite promising results in several experimental studies, no agent has made its way into clinical practice [12]. This discrepancy between experimental studies and patient treatment may be in part due to the frequent use of healthy grafts in many animal studies [13, 14]. This is not representative of the clinical situation, in which organ shortage often necessitates the use of grafts of poor quality (i.e. steatotic grafts) from extended criteria donors. Thus, the results of our study may provide a novel and clinically relevant therapeutic approach for the protection of steatotic organs, thereby increasing the pool of transplantable livers.

\section{Methods}

\section{Animals}

Male syngeneic Zucker rats (Charles River Wiga, Sulzfeld, Germany) aged 10-14 weeks were used in this study. Animals were housed in a temperature- and humidity-controlled room under a constant 12-hour light/dark cycle. Rats with a homozygote point mutation of codon 269 in the leptine receptor gene (fa/fa) develop massive obesity. In contrast, animals with a heterozygote defect of
Table 1. Study groups

\begin{tabular}{llllc}
\hline Group & Donor & Recipient & Treatment & CIT, h \\
\hline 1: fa/- veh & $\mathrm{fa} /-$ & $\mathrm{fa} /-$ & veh & 24 \\
2: fa/fa veh & $\mathrm{fa} / \mathrm{fa}$ & $\mathrm{fa} /-$ & veh & 4 \\
3: fa/fa GSH & $\mathrm{fa} / \mathrm{fa}$ & $\mathrm{fa} /-$ & GSH & 4
\end{tabular}

CIT $=$ Cold ischemia time; veh $=$ vehicle

this gene $(\mathrm{fa} /-)$ remain lean. Donors (body weight: fa/fa $478 \pm$ $63 \mathrm{~g}, \mathrm{fa} /-345 \pm 72 \mathrm{~g})$ and recipients $(\mathrm{fa} /-318 \pm 22 \mathrm{~g}$ ) had free access to water and rat chow (standard diet; Altromin, Lage, Germany). Recipient rats were fasted $12 \mathrm{~h}$ prior to donor operation and liver transplantation. All procedures were carried out in accordance with the guidelines of the Animal Welfare Act and the Guide for the Care and Use of Laboratory Animals from the National Institutes of Health. The institutional animal care and use committee of the Government of Upper Bavaria and the Ludwig-Maximilians-University (Munich, Germany) approved this project.

\section{Experimental Groups}

Male Zucker rats were randomly assigned to three groups $(\mathrm{n}=$ 5/group; table 1). In group 1 (fa/- veh) livers were explanted from lean male Zucker rats and subjected to $24 \mathrm{~h}$ of cold ischemia $\left(4^{\circ} \mathrm{C}\right)$ in University of Wisconsin solution. Orthotopic arterialized liver transplantation was then performed on lean male Zucker rats $(\mathrm{fa} /-)$. During reperfusion, $6 \mathrm{ml}$ of saline $0.9 \%$ (vehicle) were infused continuously at a rate of $3 \mathrm{ml} / \mathrm{h}$ starting $20 \mathrm{~min}$ before declamping of the portal vein and hepatic artery.

In group 2 (fa/fa veh) and 3 (fa/fa GSH) steatotic livers were explanted from obese male Zucker rats (homozygote, fa/fa). After a cold ischemia period of $4 \mathrm{~h}$ these livers were transplanted into lean Zucker rats (fa/-). This reduction in ischemic time from $24 \mathrm{~h}$ in lean organs to $4 \mathrm{~h}$ in steatotic grafts was applied based on previous studies by Amersi et al. [6]. During reperfusion, animals in group 2 received $6 \mathrm{ml}$ saline (vehicle) by continuous infusion at a rate of $3 \mathrm{ml} / \mathrm{h}$ (vehicle), whereas animals in group 3 received GSH (Tationil 600, Roche, Italy) at a concentration of $200 \mu \mathrm{mol} / \mathrm{h} / \mathrm{kg}$ dissolved in $6 \mathrm{ml}$ saline. Reperfusion time was $120 \mathrm{~min}$ in all groups.

\section{Surgical Procedures}

Donor and recipient procedures were performed under spontaneous ether inhalation. For continuous monitoring of mean arterial blood pressure and for substitution of plasma volume, left carotid artery and jugular vein were cannulated with polyethylene catheters. Body temperature was kept between 36.5 and $37.5^{\circ} \mathrm{C}$ using a heating pad. Donor livers were preserved by retrograde aortal flush with $15 \mathrm{ml}$ of University of Wisconsin solution and stored at $4^{\circ} \mathrm{C}$ for $24 \mathrm{~h}$ (group 1 ) or $4 \mathrm{~h}$ (group 2/3). Before implantation, livers were rinsed with $10 \mathrm{ml}$ of Ringer's lactate solution at $4^{\circ} \mathrm{C}$ via portal vein (hydrostatic pressure: $10 \mathrm{~cm} \mathrm{H}_{2} \mathrm{O}$ ). Orthotopic liver transplantation was performed according to the cuff technique described by Kamada and Calne [15]. Grafts were rearterialized and simultaneously reperfused via portal vein and 
hepatic artery as described by Post et al. [16]. Portal clamping times never exceeded $20 \mathrm{~min}$. Five minutes after declamping, all rats received $0.5 \mathrm{ml}$ of albumin (5\%) and $0.5 \mathrm{ml}$ of sodium bicarbonate to maintain blood pressure and physiological $\mathrm{pH}$ values. After 120 min of reperfusion, animals were sacrificed and liver weight was determined.

\section{Quantification of Bile Synthesis}

To quantify liver function during the reperfusion period, bile flow was quantified as previously described [17]. The common bile duct was cannulated with a polyethylene tube during donor surgery. Cumulative bile flow ( $\mu \mathrm{l} / \mathrm{g} / \mathrm{min})$ was calculated from total bile volume secreted between start of reperfusion and end of experiment (120 min).

\section{Determination of GSH and GSSG}

As opposed to other plasma parameters, determination of GSH (reduced GSH prior to oxidation, administered form) and GSSG (oxidized form of GSH following radical scavenging or spontaneous oxidation) in plasma requires a separate isolation procedure as described previously by Jaeschke et al. [18]. A total of $500 \mu \mathrm{l}$ of whole blood is required for determination of total GSH (GSH total = sum of GSH and GSSG). For GSSG analysis, an aliqot $(200 \mu \mathrm{l})$ of blood was mixed immediately with $200 \mu \mathrm{l}$ of $10 \mathrm{mmol} / \mathrm{l} \mathrm{N}$-ethylmaleimide (NEM) in $100 \mathrm{mmol} / \mathrm{l}$ phosphate buffer ( $\mathrm{pH}$ 6.5) containing $17.5 \mathrm{mmol} / \mathrm{l}$ EDTA. The remaining blood was centrifuged for $1 \mathrm{~min}$. An aliquot $(100 \mu \mathrm{l})$ of plasma was pipetted into $100 \mu \mathrm{l}$ sulfosalicylic acid (5\%) for determination of total GSH. To separate GSSG from NEM and NEM-GSH, an aliquot of NEM-treated plasma was passed through a SepPakC18 cartridge (Waters, Framingham, Mass., USA) followed by $1 \mathrm{ml}$ of $100 \mathrm{mmol} / \mathrm{l}$ phosphate buffer (pH 7.5). GSSG in the eluates and total GSH in acidified plasma samples was determined by an enzymatic test as described previously [19]. GSH plasma concentrations were calculated as difference between total GSH and GSSG.

\section{Plasma Collection and Storage}

Whole blood was obtained via arterial catheter line (approximately $1.5 \mathrm{ml}$ ) in microcentrifuge tubes (Microtainer, Becton Dickinson, Rutherford, N.J., USA) and centrifuged at $16,000 \mathrm{~g}$ for $15 \mathrm{~min}$ at $4^{\circ} \mathrm{C}$. Plasma was separated, placed in pyrogen-free microcentrifuge tubes, immediately frozen, and stored $\left(-80^{\circ} \mathrm{C}\right)$ until assayed for alanine aminotransferase (ALT), aspartate aminotransferase (AST) and lactate dehydrogenase (LDH).

\section{Assessment of Serum Aminotransferases}

Serum aminotransferases were used as established markers of hepatic injury. AST and ALT were measured $2 \mathrm{~h}$ after reperfusion with a kinetic UV test using a serum multiple analyzer (Olympus AU 2700) [20].

\section{Assessment of Lactate Dehydrogenase}

Systemic cellular damage was determined by assaying serum levels of LDH $2 \mathrm{~h}$ after reperfusion with a kinetic UV test using a serum multiple analyzer (Olympus AU 2700) [21].

\section{Histology}

Representative histological sections (HE staining) of fatty livers were examined $2 \mathrm{~h}$ after reperfusion.

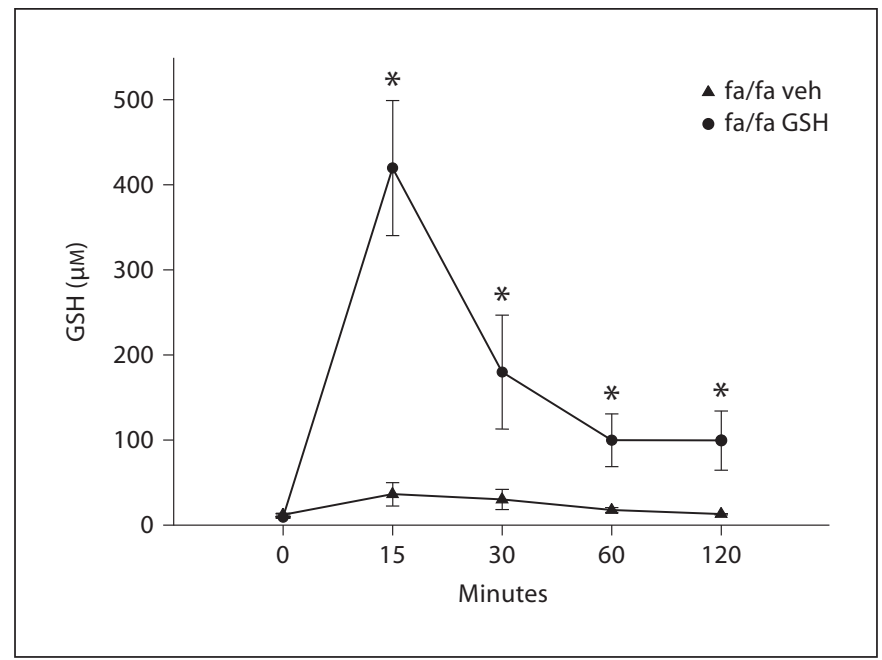

Fig. 1. Plasma GSH levels determined at time of reperfusion (0) and $15,30,60$, and $120 \mathrm{~min}$ after liver transplantation. Livers were explanted from lean (fa/-) or obese (fa/fa) rats. Prior to declamping, recipient animals received vehicle or GSH $(200 \mu \mathrm{mol} / \mathrm{kg}$ body weight/h). $n=5 /$ group. Values are presented as mean $\pm \mathrm{SD}$. Data were analyzed by one-way ANOVA. ${ }^{*} \mathrm{p}<0.05 \mathrm{vs}$. fa/- veh.

\section{Statistics}

The results are presented as mean \pm SD. One-way ANOVA followed by the Student-Newman-Keuls test or Tukey test as a post hoc test for multiple comparisons was used to determine significance of the differences between experimental means. p $<$ 0.05 was considered to be significant.

\section{Results}

\section{Plasma GSH and GSSG}

Plasma GSH was determined in rats receiving steatotic organs and treated with vehicle or GSH at time of reperfusion ( $\mathrm{t}=0 \mathrm{~min}$ ) and 15, 30, 60 and $120 \mathrm{~min}$ thereafter (fig. 1). GSH-treated rats displayed systemic peak GSH levels 15 min after reperfusion. Plasma GSH levels were significantly increased in GSH treated rats throughout the whole experiment compared to vehicle-treated animals $(\mathrm{p}<0.05)$.

Plasma GSSG levels were significantly elevated 60 and 120 min after reperfusion in GSH-treated animals compared to non-treated rats ( $\mathrm{p}<0.05$; fig. 2$)$.

\section{Plasma Aminotransferases (ALT/AST) \\ Alanine Aminotransferase}

ALT levels were significantly increased in recipients of steatotic livers compared to recipients of lean livers after 


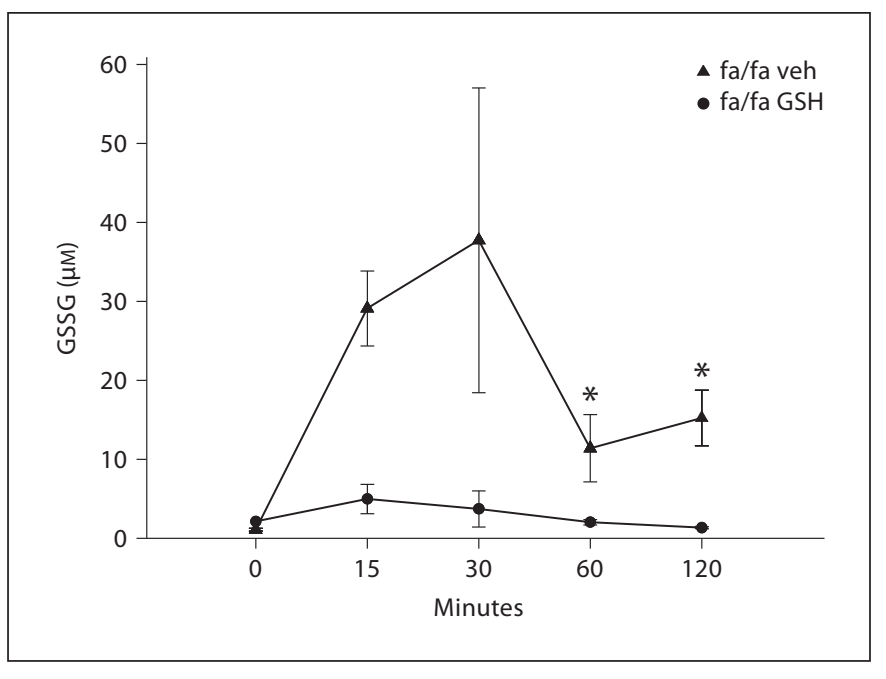

Fig. 2. Plasma GSSG levels determined at time of reperfusion (0) and $15,30,60$, and $120 \mathrm{~min}$ after liver transplantation. Livers were explanted from lean (fa/-) or obese (fa/fa) rats. Prior to declamping, recipient animals received vehicle or GSH $(200 \mu \mathrm{mol} / \mathrm{kg}$ body weight $/ h$ ). $n=5$ /group. Values are presented as mean \pm SD. Data were analyzed by one-way ANOVA. ${ }^{*} \mathrm{p}<0.05 \mathrm{vs}$. fa/- veh.

reperfusion ( $\mathrm{p}<0.05$; fig. 3 ). In recipients of steatotic grafts, administration of GSH significantly reduced plasma ALT levels compared to administration of vehicle $(\mathrm{p}<0.05)$.

\section{Aspartate Aminotransferase}

Plasma AST levels were significantly enhanced in vehicle-treated recipients of fatty livers compared to rats receiving lean organs ( $p<0.05$; fig. 4). In recipients of steatotic livers, treatment with GSH was associated with a significant reduction of AST compared to administration of vehicle $(\mathrm{p}<0.05)$.

\section{Lactate Dehydrogenase}

LDH, a non-organ-specific marker of IRI, was significantly higher in recipients of fatty livers treated with vehicle compared to recipients of lean livers following liver transplantation ( $\mathrm{p}<0.05$; fig. 5). In recipients of steatotic livers, the administration of GSH significantly reduced serum $\mathrm{LDH}$ levels compared to the administration of vehicle $(\mathrm{p}<0.05)$.

\section{Bile Flow}

Two hours after reperfusion, cumulative bile flow (a sensitive marker of liver function) was significantly diminished in steatotic livers receiving vehicle compared to lean grafts ( $p<0.05$; fig. 6). GSH treatment, however, significantly increased bile flow in steatotic grafts compared to treatment with vehicle $(\mathrm{p}<0.05)$.

\section{Histology}

Histological sections (HE staining) demonstrated a $50-80 \%$ degree of steatosis (data not shown). Two hours after liver transplantation, no alterations between the groups and due to IRI were evident.

\section{Discussion}

The critical organ shortage for liver transplantation has resulted in the routine acceptance of steatotic organs. In the general population, steatosis occurs with a prevalence of $13-50 \%[1,2]$. Steatotic livers substantially contribute to the donor pool, thereby partially compensating for organ shortage. Clinical and experimental studies have shown that steatotic livers are more susceptible to IRI than lean livers [3, 4, 22-25]. This results in increased plasma aminotransferases and diminished liver function in the early postoperative phase following transplantation $[3,4]$. Other risk factors, including cold ischemia time and donor age $[26,27]$, have been shown to exhibit additive detrimental effects on organ function and survival following liver transplantation. Steatosis is a particularly relevant risk factor for IRI due to its increasing prevalence. Moreover, in contrast to risk factors such as cold ischemia time, steatosis cannot be affected by changing allocation procedures. Nonetheless, future experimental studies should consider other risk factors beside steatosis to better mimic the clinical situation.

Due to the clinical importance of steatosis for outcome following liver transplantation, animal models of steatosis have been established [2]. Genetically obese Zucker rats have been shown to develop liver steatosis mimicking non-alcoholic fatty liver disease $[2,28]$. Interestingly, the pathophysiological mechanisms associated with reperfusion injury after orthotopic liver transplantation have been shown to be different between lean and steatotic Zucker rats [11]. Lean rats tend to develop hepatic apoptosis following ischemia-reperfusion whereas necrosis is the predominant form of cell death in steatotic organs [11]. Moreover, steatotic livers exhibit an increased production of reactive oxygen species (ROS) following warm and cold ischemia and reperfusion $[5,29]$. These results collectively suggest that steatosis has to be considered when investigating new therapeutic strategies in liver transplantation. 


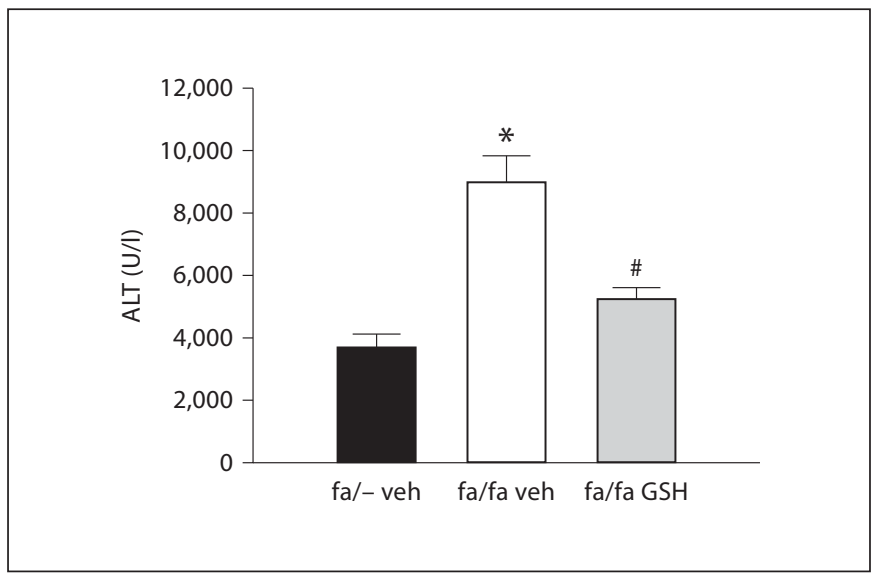

Fig. 3. Serum ALT levels determined $2 \mathrm{~h}$ after liver transplantation. Livers were explanted from lean $(\mathrm{fa} /-)$ or obese $(\mathrm{fa} / \mathrm{fa})$ rats. Prior to declamping, recipient animals received vehicle or GSH (200 $\mu \mathrm{mol} / \mathrm{kg}$ body weight $/ \mathrm{h}) . \mathrm{n}=5 /$ group. Values are presented as mean \pm SD. Data were analyzed by one-way ANOVA. ${ }^{*} \mathrm{p}<$ 0.05 vs. fa/- veh; ${ }^{*} \mathrm{p}<0.05$ vs. fa/fa veh.

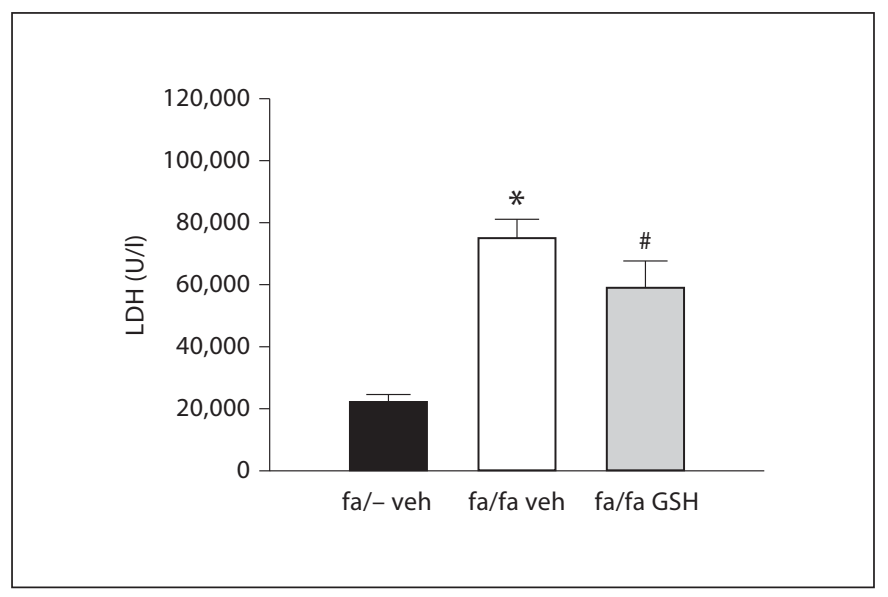

Fig. 5. Serum LDH levels determined $2 \mathrm{~h}$ after liver transplantation. Livers were explanted from lean $(\mathrm{fa} /-)$ or obese (fa/fa) rats. Prior to declamping, recipient animals received vehicle or GSH ( $200 \mu \mathrm{mol} / \mathrm{kg}$ body weight/h). $\mathrm{n}=5 /$ group. Values are presented as mean $\pm \mathrm{SD}$. Data were analyzed by one-way ANOVA. ${ }^{*} \mathrm{p}<$ 0.05 vs. fa/- veh; ${ }^{*}$ p $<0.05$ vs. fa/fa veh.

The present data indicate that steatotic livers harvested from genetically obese Zucker rats develop significantly more organ injury compared to lean allografts. Interestingly, increased organ injury following transplantation of fatty organs was evident despite a reduction in cold ischemia time from $24 \mathrm{~h}$ in lean to $4 \mathrm{~h}$ in steatotic

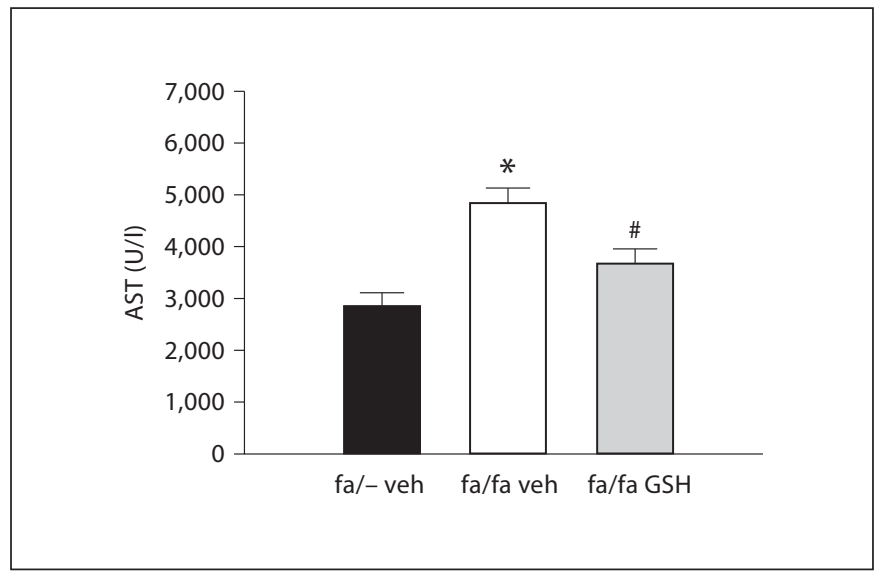

Fig. 4. Serum AST levels determined $2 \mathrm{~h}$ after liver transplantation. Livers were explanted from lean $(\mathrm{fa} /-)$ or obese (fa/fa) rats. Prior to declamping, recipient animals received vehicle or GSH (200 $\mu \mathrm{mol} / \mathrm{kg}$ body weight/h). $\mathrm{n}=5 /$ group. Values are presented as mean $\pm \mathrm{SD}$. Data were analyzed by one-way ANOVA. ${ }^{*} \mathrm{p}<$ 0.05 vs. fa/- veh; ${ }^{*} \mathrm{p}<0.05$ vs. fa/fa veh.

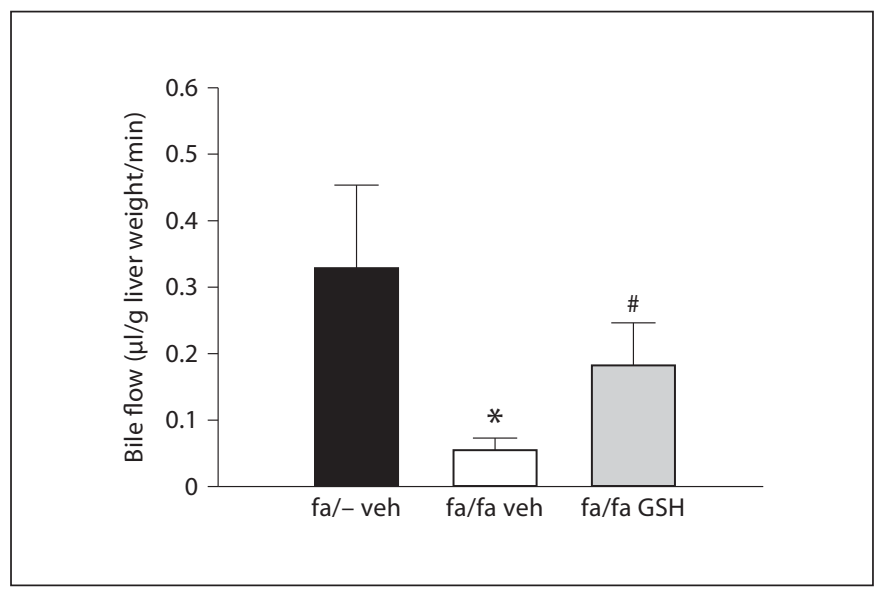

Fig. 6. Bile flow ( $\mu \mathrm{l} / \mathrm{g}$ liver weight/min) during $2 \mathrm{~h}$ of reperfusion. Livers were explanted from lean $(\mathrm{fa} /-)$ or obese (fa/fa) rats. Prior to declamping, recipient animals received vehicle or GSH (200 $\mu \mathrm{mol} / \mathrm{kg}$ body weight $/ \mathrm{h}) . \mathrm{n}=5 /$ group. Values are presented as mean \pm SD. Data were analyzed by one-way ANOVA. ${ }^{*} \mathrm{p}<0.05$ vs. fa/- veh; ${ }^{\#}$ p $<0.05$ vs. fa/fa veh.

organs. These findings are supported by previous results demonstrating increased organ damage and diminished organ function in fatty compared to lean organs following IRI [ 30,31$]$. Cold ischemia time of $24 \mathrm{~h}$ for healthy donor organs was based on previous studies, demonstrating significant IRI in recipient animals $[13,17]$. For stea- 
totic organs, cold ischemia time was substantially reduced to avoid deleterious organ damage [7].

In healthy organs, continuous administration of GSH protects liver tissue from reperfusion injury after warm and cold ischemia $[9,10,32]$. Endogenous GSH is concentrated in the intracellular space [33]. It reacts with oxidants released during reperfusion resulting in formation of oxidized GSH (GSSG) [34]. Its effect on ischemia-reperfusion injury in steatotic grafts, however, remains unknown. In the present study, GSH was administered at a dosage of $200 \mu \mathrm{mol} / \mathrm{kg}$ body weight/h to provide steatotic organs with supraphysiological GSH levels. This dosage has been shown to ameliorate IRI following transplantation of lean liver allografts [30] and resulted in peak plasma GSH levels below the limit of toxicity in humans $(500 \mu \mathrm{M})$ [35]. As has been found in lean organs, the administration of GSH at the beginning of reperfusion significantly decreased early liver injury (plasma aminotransferase levels) and ameliorated liver function (bile flow) in steatotic grafts.

Determination of the exact mechanisms which are responsible for the beneficial effects of GSH in steatotic organs was beyond the scope of our study. Nonetheless, GSSG levels, the oxidized form of GSH, were increased in GSH-treated recipients of fatty grafts. This suggests that intravenously applied GSH was mostly oxidized. Oxidation of GSH is known to be associated with detoxification of detrimental ROS $[36,37]$. In addition, the metabolism of GSH produces glycine [35], which also has protective properties against liver reperfusion injury [14]. It has been proposed that the GSH-induced reduction of ROS is associated with improved sinusoidal perfusion, significant decrease of leukocytes sticking to sinusoids, as well as prevention of sinus endothelial cell injury in lean organs $[10,32]$. Steatotic livers are characterized by decreased sinusoidal blood flow due to swollen hepatocytes which may induce chronic hypoxia [38] and ATP depletion [39]. Moreover, ischemia-reperfusion injury in fatty organs is associated with enhanced leukocyte adhesion and microcirculatory failure, potentially based on an increased ROS release $[23,25,29,40]$. These studies collectively suggest that GSH may also decrease ROS in fatty organs following cold storage and transplantation, thereby improving microcirculation and preventing endothelial damage, at least during the early reperfusion period which was of particular interest in this study.

In summary, the present study demonstrated increased IRI in transplanted, fatty allografts. Continuous postischemic infusion of GSH, a ROS scavenger, ameliorated early hepatocellular injury and improved liver function in fatty organs. The protective effects of GSH were associated with an increase in oxidized plasma GSSG, indicating detoxification of ROS by GSH. Since no severe side effects of GSH administration have been reported, this peptide has significant potential to be a useful and safe means of improving the function of steatotic organs following liver transplantation in humans. A prospective randomized trial is required to verify these promising experimental results in the clinical arena.

\section{Acknowledgment}

This work was supported in part by a grant from the Deutsche Forschungsgemeinschaft (DFG 440-Scha 857/1-1 and Scha 857/ 1-2).

\section{References}

1 Marsman WA, Wiesner RH, Rodriguez L, Batts KP, Porayko MK, Hay JE, et al: Use of fatty donor liver is associated with diminished early patient and graft survival. Transplantation 1996;62:1246-1251.

2 Koneru B, Dikdan G: Hepatic steatosis and liver transplantation current clinical and experimental perspectives. Transplantation 2002;73:325-330

3 McCormack L, Petrowsky H, Jochum W, Mullhaupt B, Weber M, Clavien PA: Use of severely steatotic grafts in liver transplantation: a matched case-control study. Ann Surg 2007;246:940-946.
4 Angele MK, Rentsch M, Hartl WH, Wittmann B, Graeb C, Jauch KW, et al: Effect of graft steatosis on liver function and organ survival after liver transplantation. Am J Surg 2008; 195:214-220.

5 Soltys K, Dikdan G, Koneru B: Oxidative stress in fatty livers of obese Zucker rats: rapid amelioration and improved tolerance to warm ischemia with tocopherol. Hepatology 2001;34:13-18.

6 Amersi F, Shen XD, Moore C, Melinek J, Busuttil RW, Kupiec-Weglinski JW, et al: Fibronectin-alpha 4 beta 1 integrin-mediated blockade protects genetically fat Zucker rat livers from ischemia/reperfusion injury. Am J Pathol 2003;162:1229-1239.
7 Bilzer M, Paumgartner G, Gerbes AL: GSH protects the rat liver against reperfusion injury after hypothermic preservation. Gastroenterology 1999;117:200-210.

8 Jaeschke H: Mechanisms of liver injury. II. Mechanisms of neutrophil-induced liver cell injury during hepatic ischemia-reperfusion and other acute inflammatory conditions. Am J Physiol Gastrointest Liver Physiol 2006;290:G1083-G1088.

9 Bilzer M, Baron A, Schauer R, Steib C, Ebensberger S, Gerbes AL: GSH treatment protects the rat liver against injury after warm ischemia and Kupffer cell activation. Digestion 2002;66:49-57. 
10 Schauer RJ, Kalmuk S, Gerbes AL, Leiderer $\mathrm{R}$, Meissner H, Schildberg FW, et al: Intravenous administration of GSH protects parenchymal and non-parenchymal liver cells against reperfusion injury following rat liver transplantation. World J Gastroenterol 2004; 10:864-870.

11 Selzner M, Rudiger HA, Sindram D, Madden J, Clavien PA: Mechanisms of ischemic injury are different in the steatotic and normal rat liver. Hepatology 2000;32:1280-1288.

12 Amersi F, Farmer DG, Shaw GD, Kato H, Coito AJ, Kaldas F, et al: P-selectin glycoprotein ligand-1 (rPSGL-Ig)-mediated blockade of CD62 selectin molecules protects rat steatotic liver grafts from ischemia/reperfusion injury. Am J Transplant 2002;2:600-608.

13 Bradham CA, Schemmer P, Stachlewitz RF, Thurman RG, Brenner DA: Activation of nuclear factor-kappaB during orthotopic liver transplantation in rats is protective and does not require Kupffer cells. Liver Transpl Surg 1999;5:282-293.

14 Schemmer P, Bradford BU, Rose ML, Bunzendahl H, Raleigh JA, Lemasters JJ, et al: Intravenous glycine improves survival in rat liver transplantation. Am J Physiol 1999; 276:G924-G932.

15 Kamada N, Calne RY: A surgical experience with 530 liver transplants in the rat. Surgery 1983;93:64-69.

16 Post S, Gonzalez AP, Palma P, Rentsch M, Stiehl A, Menger MD: Assessment of hepatic phagocytic activity by in vivo microscopy after liver transplantation in the rat. Hepatology 1992;16:803-809.

17 Schauer RJ, Bilzer M, Kalmuk S, Gerbes AL, Leiderer R, Schildberg FW, et al: Microcirculatory failure after rat liver transplantation is related to Kupffer cell-derived oxidant stress but not involved in early graft dysfunction. Transplantation 2001;72:1692-1699.

18 Jaeschke H, Mitchell JR: Use of isolated perfused organs in hypoxia and ischemia/reperfusion oxidant stress. Methods Enzymol 1990; 186:752-759.

19 Tietze F: Enzymic method for quantitative determination of nanogram amounts of total and oxidized GSH: applications to mammalian blood and other tissues. Anal Biochem 1969;27:502-522.
20 Bergmeyer HU, Horder M, Rej R: International Federation of Clinical Chemistry (IFCC) Scientific Committee, Analytical Section: approved recommendation (1985) on IFCC methods for the measurement of catalytic concentration of enzymes. Part 3. IFCC method for alanine aminotransferase (L-alanine: 2-oxoglutarate aminotransferase, EC 2.6.1.2). J Clin Chem Clin Biochem 1986;24:481-495.

21 Bais R, Philcox M: Approved recommendation on IFCC methods for the measurement of catalytic concentration of enzymes. Part 8 IFCC Method for Lactate Dehydrogenase (LLactate: NAD+Oxidoreductase, EC 1.1.1.27) International Federation of Clinical Chemistry (IFCC). Eur J Clin Chem Clin Biochem 1994;32:639-655.

22 Reddy MC, Koneru B, Soni S, Patel D: 31P nuclear magnetic resonance study of phospholipids in ischemia/reperfusion injury in a rat fatty liver model. Transplantation 1996 61:1151-1155.

23 Teramoto K, Bowers JL, Kruskal JB, Clouse ME: Hepatic microcirculatory changes after reperfusion in fatty and normal liver transplantation in the rat. Transplantation 1993; 56:1076-1082.

24 Selzner M, Clavien PA: Fatty liver in liver transplantation and surgery. Semin Liver Dis 2001;21:105-113.

25 Sun CK, Zhang XY, Zimmermann A, Davis G, Wheatley AM: Effect of ischemia-reperfusion injury on the microcirculation of the steatotic liver of the Zucker rat. Transplantation 2001;72:1625-1631

26 Burroughs AK, Sabin CA, Rolles K, Delvart V, Karam V, Buckels J, et al: 3-month and 12-month mortality after first liver transplant in adults in Europe: predictive models for outcome. Lancet 2006;367:225-232.

27 Afonso RC, Hidalgo R, Paes AT, Zurstrassen MP, Fonseca LE, Pandullo FL, et al: Impact of cumulative risk factors for expanded criteria donors on early survival after liver transplantation. Transplant Proc 2008;40: 800-801.

28 Phillips MS, Liu Q, Hammond HA, Dugan V, Hey PJ, Caskey CJ, et al: Leptin receptor missense mutation in the fatty Zucker rat. Nat Genet 1996;13:18-19.

29 Nardo B, Caraceni P, Pasini P, Domenical $\mathrm{M}$, Catena F, Cavallari G, et al: Increased generation of reactive oxygen species in isolated rat fatty liver during postischemic reoxygenation. Transplantation 2001;71:18161820 .
30 Lehmann TG, Wheeler MD, Schwabe RF, Connor HD, Schoonhoven R, Bunzendahl $\mathrm{H}$, et al: Gene delivery of $\mathrm{Cu} / \mathrm{Zn}$-superoxide dismutase improves graft function after transplantation of fatty livers in the rat. Hepatology 2000;32:1255-1264.

31 Choi S, Noh J, Hirose R, Ferell L, Bedolli M, Roberts JP, et al: Mild hypothermia provides significant protection against ischemia/reperfusion injury in livers of obese and lean rats. Ann Surg 2005;241:470-476.

32 Schauer RJ, Gerbes AL, Vonier D, Meissner $\mathrm{H}$, Michl P, Leiderer R, et al: GSH protects the rat liver against reperfusion injury after prolonged warm ischemia. Ann Surg 2004; 239:220-231.

33 Lauterburg BH, Adams JD, Mitchell JR: Hepatic GSH homeostasis in the rat: efflux accounts for GSH turnover. Hepatology 1984; 4:586-590.

34 Bilzer M, Lauterburg BH: GSH metabolism in activated human neutrophils: stimulation of GSH synthesis and consumption of GSH by reactive oxygen species. Eur J Clin Invest 1991;21:316-322.

35 Aebi S, Assereto R, Lauterburg BH: Highdose intravenous GSH in man: pharmacokinetics and effects on cyst(e)ine in plasma and urine. Eur J Clin Invest 1991;21:103-110.

36 Akerboom TP, Gartner M, Sies H: Cellular hydroperoxide metabolism: the roles of GSH peroxidases and of catalase in liver. Bull Eur Physiopathol Respir 1981;17(suppl):221-227.

37 Dickinson DA, Forman HJ: GSH in defense and signaling: lessons from a small thiol. Ann NY Acad Sci 2002;973:488-504.

38 Seifalian AM, Piasecki C, Agarwal A, Davidson BR: The effect of graded steatosis on flow in the hepatic parenchymal microcirculation. Transplantation 1999;68:780-784.

39 Serkova NJ, Jackman M, Brown JL, Liu T, Hirose R, Roberts JP, et al: Metabolic profiling of livers and blood from obese Zucker rats. J Hepatol 2006;44:956-962.

40 Yamagami K, Hutter J, Yamamoto Y, Schauer RJ, Enders G, Leiderer R, et al: Synergistic effects of brain death and liver steatosis on the hepatic microcirculation. Transplantation 2005;80:500-505. 